\title{
Bifunctional Role of Thiosalicylic Acid in the Synthesis of Silver Nanoparticles
}

\author{
Ramasamy Indumathy, Kalarical Janardhanan Sreeram, Muralidharan Sriranjani, Cheravathoor \\ Poulose Aby, Balachandran Unni Nair
}

Chemical Laboratory, Central Leather Research Institute, Council of Scientific and Industrial Research, Chennai, India. Email: kjsreeram@clri.res.in

Received July $30^{\text {th }}, 2010$; revised October $19^{\text {th }}, 2010$; accepted October $30^{\text {th }}, 2010$.

\begin{abstract}
Conventional synthesis of silver nanoparticles employs a reducing agent and a capping agent. Surfactants are effective capping agents as they prevent the aggregation of nanoparticles during storage and use. However, the biocompatibility of several of the surfactants is questionable. In this report, the use of thiosalicylic acid as both reducing and capping agent is reported. Compared to conventional synthesis, this methodology requires higher temperature for synthesis, which then is expected to result in aggregates of larger size. The ability of three different synthesis methodologies direct heating, photochemical and microwave dielectric treatment were evaluated and assessed on the basis of the size, size distribution and stability of the particles. Microwave irradiation was found to be most suitable for achieving particles with a hydrodynamic diameter of $10 \mathrm{~nm}$. Our studies indicate that -COO-group is involved in the reduction of Ag+ and-SH group of TSA is involved in the capping of the nanoparticles.
\end{abstract}

Keywords: Silver Nanoparticles, Microwave Dielectric Heating, Reducing cum Capping Agent, Thiosalicylic Acid, Photon Correlation Spectroscopy, Zeta Potential

\section{Introduction}

Metal nanostructures owing to their unique physical, chemical, electrical and optical properties have acquired immense attention from the researcher's point of view. The potential application of the nanostructures can be tailored by controlling their size, shape, composition, and crystalline character [1-3]. Among the noble metals, silver nanoparticles has gained more importance due to its application in catalysis, photonics, real time optical sensors, printed electronics, surface enhanced Raman scattering and as anti-microbial agents [4-9]. They also find applications in the field of biology as antibacterials, in DNA sequencing etc., because they are biocompatible and possess less cytotoxicity. The synthesis of monodispersed and nanometer sized particles remains as great task due to which the research in the field of synthetic methodology of nanomaterials is being held as an endless endeavor. Recent reports on nanomaterial synthesis reveal that the size,

Funding support from CSIR through the NWP0035 - Nanomaterials and nanodevices for applications in health and diseases as well as the OLP-57 EMPOWER project on collagen stabilization using functionalized nanoparticles. shape and stability of the nanoparticles depend strongly on the specificity on the method and experimental conditions implemented for their synthesis. Thus, new synthetic strategies are frequently reported in the literature. A variety of approaches have been reported in literature for synthesizing silver nanoparticles. This includes chemical reduction in aqueous media with or without stabilizers $[10,11]$, formation in micro emulsion, thermal decomposition, use of Langmuir-Blodgett films, biosynthesis using microorganisms, fungus and the use of liquid crystalline phases made of surfactant aggregates, etc [12-21].

The solution chemistry relating to the synthesis of silver nanoparticles has predominantly been those involving reduction of silver colloidal systems with reductants. Sodium citrate and sodium borohydride are often used as the most effective reducing agents [22]. To circumvent the problem of aggregation, researchers have been employing surface active agents or capping agents in addition to reducing agents [23,24]. Use of organic compounds, alkanethiols and bisulfides as well as polymers has been described, the most important ones being surfactants and carboxylic acids [25]. Amongst the several 
approaches developed for the nanoparticle synthesis, photo activation [26,27] and microwave [28-30] assisted techniques serves as a simple and straight forward methods for the rapid and size controlled synthesis of metal nanoparticles.

In this work, a combination approach based on classical colloidal chemistry (for reduction) and modern nanotechnology (for stabilization) has been employed to synthesize stable silver nanoparticles. We present here a thiosalicylic acid derivatized system, wherein the TSA under appropriate mole ratios, serves a dual role of both as a reducing and capping agent for the generated silver nanoparticles. The rate of silver nanoparticles formation and their stability is evaluated by adopting three different synthetic methodologies namely, thermal, photochemical, and microwave dielectric heating.

\section{Materials and Methods}

\subsection{Materials}

Silver nitrate $\left(\mathrm{AgNO}_{3}\right)(99.9 \%$ purity) and thiosalicylic acid (TSA) (98.0\% purity) were sourced from M/s. SD Fine Chemicals India Pvt Ltd. All chemicals were used as received and de-ionized water was used throughout the work.

\subsection{Particle Synthesis}

Three different synthetic methodologies were adopted in order to compare the rate of formation and stability of the nanoparticles. The photochemical and microwave treatment were done only for the samples of definite mole ratio which showed very slow rate of nanoparticle formation at thermal conditions. Typically, $0.01 \mathrm{M}$ aqueous solutions of TSA and $\mathrm{AgNO}_{3}$ were prepared and stored under dark conditions. To a known volume of TSA, $\mathrm{AgNO}_{3}$ solution was added drop wise under constant stirring, such that after the completion of the addition, the resultant solution had a TSA: $\mathrm{AgNO}_{3}$ concentration of 1:0.25 to $1: 2$. The solution was kept stirring for $10 \mathrm{~min}$, during which time the silver particles were formed. All reactions were carried out at room temperature. For the photochemical approach, appropriate amounts of TSA and $\mathrm{AgNO}_{3}$ were taken in a quartz cuvette of $3 \mathrm{ml}$ capacity. The cuvette was placed at distance of $5 \mathrm{~cm}$ from the light source and was irradiated for about $20 \mathrm{~min}$. For microwave heating, the reactions were carried out in a domestic microwave oven with an operating frequency of $2450 \mathrm{MHz}$, where TSA and $\mathrm{AgNO}_{3}$ of different mole ratios were mixed at ambient temperature and subjected to microwave heating for about $30 \mathrm{sec}$. For obtaining the silver nanoparticles in solid state, the solution was centrifuged at $10,000 \mathrm{rpm}$ for $10 \mathrm{~min}$.

\subsection{Experimental Techniques}

UV-vis absorption spectrum of the silver containing solution was measured using a Lambda 35 UV-visible spectrophotometer from M/s. Perkin Elmer Ltd., after appropriate dilution. The size of the nanoparticles was determined using transmission electron microscopy (TEM) on a JEOL 3010 electron microscope under an acceleration voltage of $300 \mathrm{kV}$. For this, a drop of silver colloid was placed on a holey carbon film supported by a 300 mesh copper grid and the solvent allowed to evaporate. The oxidation state of the silver in the dried sample was determined by using X-ray Photoelectron Spectroscopy (XPS). The infrared (IR) spectrum was recorded on a Perkin Elmer RX-1 model FT-IR spectrophotometer on the sample pelletized with $\mathrm{KBr}$ powder. Thermo Gravimetric Analysis (TGA) was performed using a universal V 3.9 A equipment from M/s. TA instrument. These studies used ramp setting of $10^{\circ} \mathrm{C} / \mathrm{min}$ from $32^{\circ}$ to $1000^{\circ} \mathrm{C}$. Light scattering measurements were carried out at $90^{\circ}$ on a photon correlation spectrometer (PCS) from Malvern Instruments, Zetasizer 3000 HSA equipped with a digital autocorrelator. The solution was used as such for the measurement of particle size and zeta potentials. The electrophoretic mobility measurements were performed using the same setting equipped with a platinum electrode. The electrode was cleaned for $10 \mathrm{~min}$ in an ultrasonic bath prior to each measurement and pre-equilibrated for $2 \mathrm{~min}$ in an aliquot of the sample. The particle size (hydrodynamic diameter) and the particle size distribution (through CONTIN analysis) were obtained directly from the instrument. The size and size distribution of the particles were measured for the AgNPs obtained after centrifugation and drying and then re-dispersion of the silver particles in water through sonication.

\section{Results and Discussion}

The thiosalicylic acid mediated synthesis of silver nanoparticles is a slow reaction (in the order of minutes), and hence the reaction has been monitored through UV-visible spectroscopy. Nanoparticle formation is favored only by deliberate addition of $\mathrm{AgNO}_{3}$ to the aqueous solution of TSA and also for certain mole ratios, namely, 1:0.25 to 1:2 (TSA: $\mathrm{AgNO}_{3}$ ), beyond which it results in either aggregation or precipitation. The formation of metal colloids through the reduction of their ions in solution involves two stages: nucleation and growth, whose relative rates determine the size distributions of colloids. These two processes can be affected by many factors, amongst which the concentration of the two reactants plays an important role. Originally described by Ritchie [31], surface plasmon resonance is an important optical property of nanoparticles and is described as coherent fluctuations 
in electron density occurring at a "free electron" metal/ dielectric interface. "Free electron" metals are those metals, which have lone electron in valence shell such as $\mathrm{Au}$, $\mathrm{Ag}, \mathrm{Al}$ and $\mathrm{Cu}$. The surface plasmon absorptions are responsible for "red" and "yellow" colors of the gold and silver colloids, respectively. In general bare silver nanoparticles exhibit surface plasmon resonance in the region 380-400 nm [32]. The UV absorption spectrum for silver nanoparticle formation for different mole ratios of $\mathrm{AgNO}_{3}$ and TSA is presented in Figure 1. In the present systems, we observed bands in the range 420$432 \mathrm{~nm}$, which matches with earlier observations [33]. A shift in the Surface Plasmon Resonance to higher wavelength has been attributed to an increase in the size of the nanoparticles [34].

Photo irradiation of the mixture, namely, $\mathrm{AgNO}_{3}$ and TSA, resulted in a yellowish brown color solution. Theformation of the nanoparticle was corroborated by UV-visible spectrometry. Figure 2 shows the UV absorption spectrum for the AgNPs obtained by photo irra-

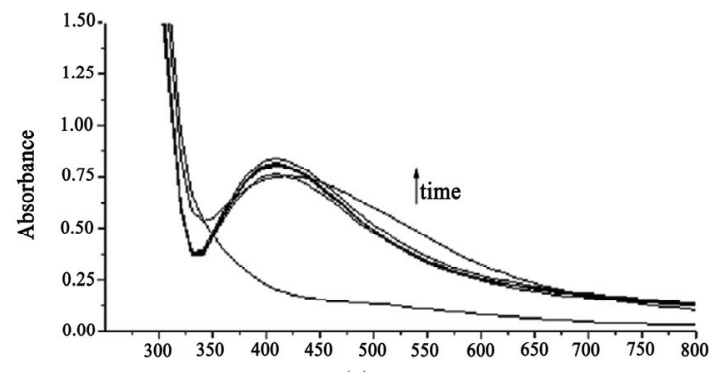

(a)

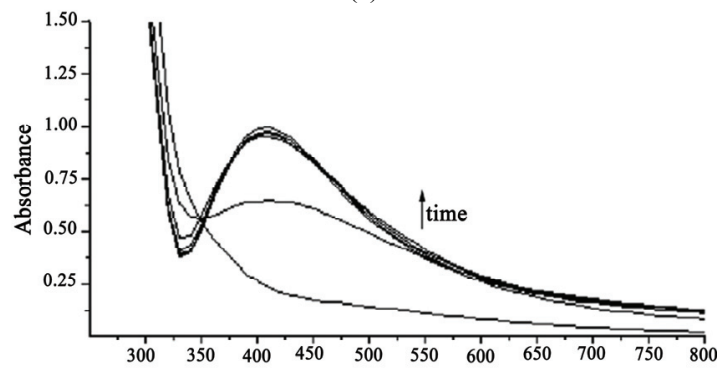

(b)

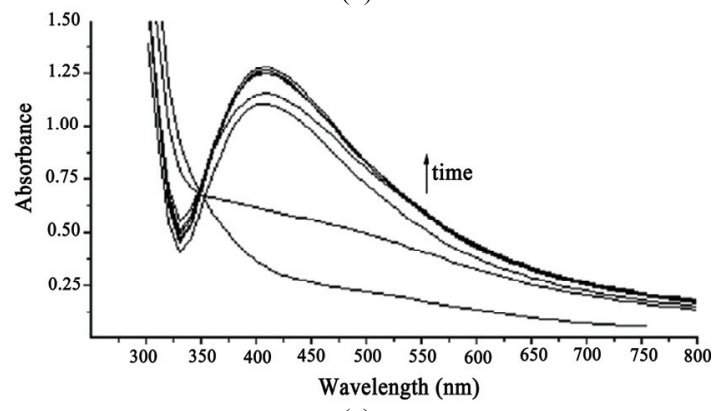

(c)

Figure 1. Absorption spectra of the TSA-Ag solution with different mole ratios (TSA: $\mathbf{A g N O}_{3}$ ) prepared at room temperature where (a) 1:0.25; (b) 1:0.5 and (c) 1:2.0.

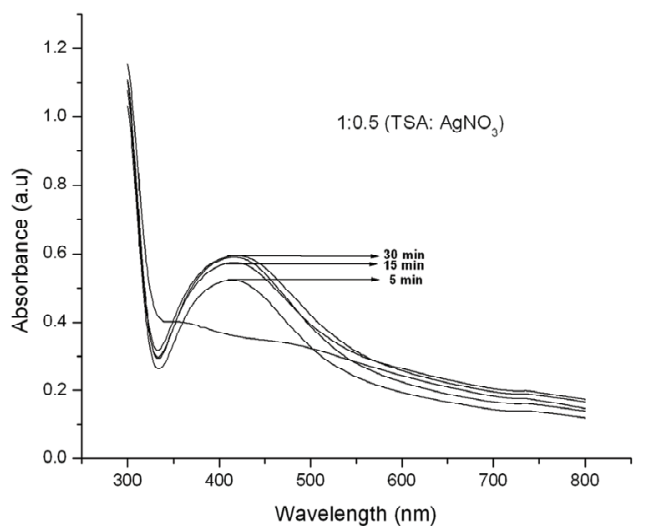

Figure 2. UV-Vis spectra of the TSA-Ag solution prepared by photochemical approach at different time intervals.

diation at different time intervals.

Almost monodispersed AgNPs were obtained. By this method in addition to the excellent reproducibility, photochemical method is more advantageous as it results in the formation of nanoparticles at a faster rate when compared to that of the reaction carried out at ambient temperature. No significant difference between the absorption spectra shapes existed after a particular duration of time, i.e., after $20 \mathrm{~min}$, indicating no difference in the morphology and size of the formed silver particles.

Microwave dielectric heating ended up with a brown color solution. The reaction was found to be highly expeditious, in order of about 10 seconds. Figure 3 shows the UV absorption spectrum for the AgNPs obtained by microwave irradiation at different time intervals.

This observation can be attributed to the fact that the high penetration depth of microwaves leads to fast and uniform heating which results in minimization of thermal gradients. The process of particle formation was also

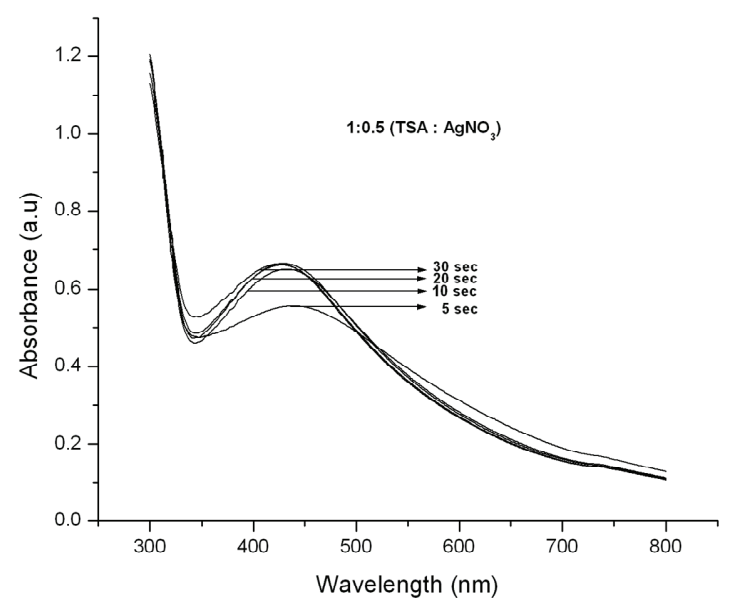

Figure 3. UV-Vis spectra of the TSA-Ag solution prepared by microwave heating at different time intervals for the sample with 1:0.5 mole ratios of TSA and $\mathrm{AgNO}_{3}$. 
monitored by UV-visible spectra for the nanoparicles synthesized by microwave irradiation. The absence of a band at $275 \mathrm{~nm}$ indicates the absence of $\mathrm{Ag}^{+} / \mathrm{Ag}^{2+}$ ions. The presence of well characterized absorption peak at $420 \mathrm{~nm}$ is an indicative of the effectiveness of thiosalicylic acid as a reductant, similar to our earlier observations. The narrow peaks observed give indirect evidence to the ability of the reductant to function as a stabilizer of the nanoparticles against aggregation.

Transmission electron microscopic (TEM) analysis of the silver nanoparticles obtained by thermal, photochemical and microwave irradiation is presented in Figure 4. TEM images clearly signifies that the particles obtained by all the methodologies were more or less uniform in size and have a spherical morphology, with an average diameter of $6 \mathrm{~nm}$. The formation of uniform and well-shaped particles can be accounted as the consequence of balance between stabilization and crystalgrowth. The effective separation of the nanoparticles in the TEM image clearly indicates that the nanoparticles with well passivated surface by the TSA molecules. The same has been further confirmed from the lattice image HRTEM and SAED pattern presented in Figure 5. It is clear from the SAED pattern that the silver nanoparticles are single crystalline and have been indexed on the basis of $f c c$ structure of silver. The diffraction spots marked by circles correspond to the (111) reflections and (200) reflections. The presence of uniformly sized nanoparticles of silver, with no prominent aggregation is indicative of the ability of thiosalicylic acid to function as a stabilizer of the nanoparticles.

The particle size for the silver nanoparticles in solution synthesized by microwave irradiation, has been measured using Photon Correlation Spectroscopy and by employing Mie theory. The number average diameter was found to be $2 \mathrm{~nm}$ (using CONTIN method of analysis). It was observed that the particle size distribution was narrow, lying between $1 \mathrm{~nm}$ and $8 \mathrm{~nm}$. The polydispersity index was 0.53 , indicating a predominant monodisperse character for the generated silver nanoparticles. In order to compare the particle size characteristics of the AgNPs, the particle size distribution for the regenerated AgNPs were also studied. This was done by centrifugation of the reaction mixture followed by washing of the particles in water and subsequent drying. The dried silver nanoparticles were then redispersed in water and the particle size and particle size distribution pattern was analyzed. The number average diameter of the particles was $7 \mathrm{~nm}$ and the polydispersity index was 0.59 . It is obvious from the particle size measurements, that there is no or minimal variations in the particle size and its distribution between original and regenerated AgNPs prepared by microwave irradiation method.

Owing to a higher surface energy, the nanoparticles tend to aggregate with time. The stability of nanoparticles against aggregation is a major requirement for several applications, most importantly in medicine. Zeta potential measurements are indicative of the stability of the particles to remain discrete in a given medium. The zeta potential values for the silver nanoparticles in the reaction medium after the completion of reaction under thermal, photochemical and microwave heating were found to be $-24.8 \pm 1.6 \mathrm{mV},-26.7 \pm 1.6 \mathrm{mV}$, and $-41.5 \pm$ $1.6 \mathrm{mV}$ respectively. A large negative zeta potential is a measure of the stability of the nanoparticles against aggregation. From the zeta potential values, it is evident that the nanoparticles prepared by the three methods were found to stable. The enhanced stability offered by the microwave irradiation may be due to the complete reduction of metal salt along with effective capping offered by TSA under microwave heating. The stability of the nanoparticles against aggregation needs to be correlated to the possible capping that the medium provide.

In order to understand the role of the medium (thio-

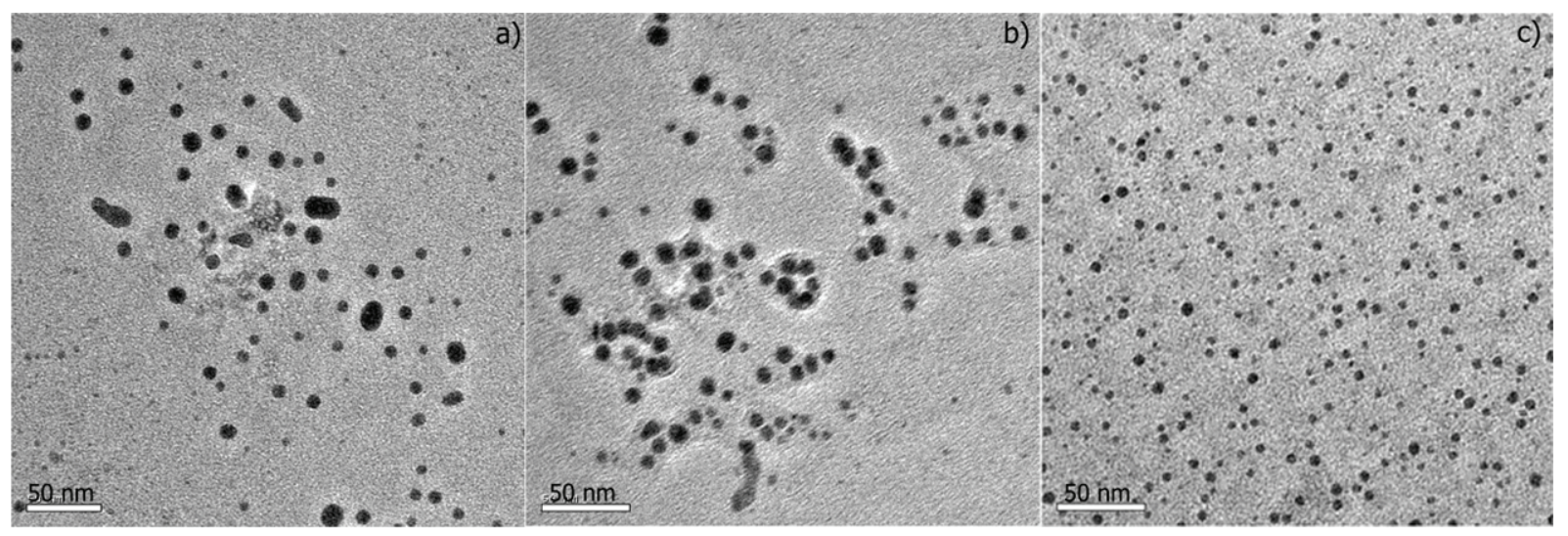

Figure 4. Transmission electron micrograph of silver nanoparticles synthesized through (a) thermal treatment, (b) photo irradiation and (c) microwave dielectric heating. 


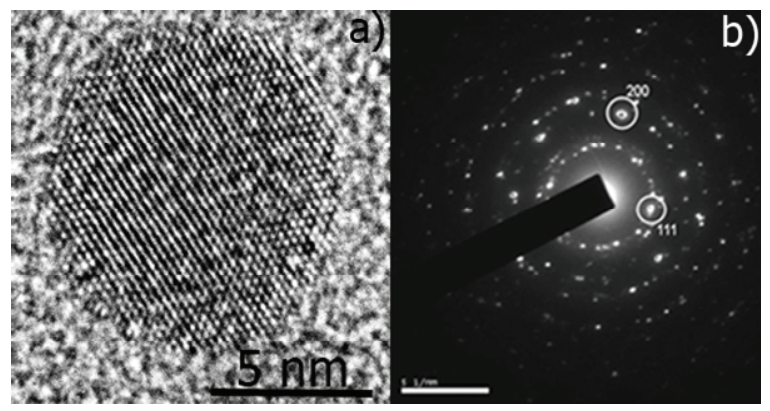

Figure 5. (a) High resolution transmission electron micrograph and (b) SAED pattern of the silver nanoparticles prepared through microwave dielectric heating.

salicylic acid) in providing stability to silver nanoparticles, a FT-IR spectroscopic measurement of the dry silver nanoparticles was carried out. FT-IR spectrum recorded for TSA derivatized AgNPs and free TSA is shown in Figure 6. In the FT-IR spectrum of AgNPs, the appearance of the strong band at $1633 \mathrm{~cm}^{-1}$ and comparatively a weak band at $1461 \mathrm{~cm}^{-1}$ can be assigned to the symmetric and antisymmetric stretching vibration of $\mathrm{COO}^{-}$from the thiosalicylate. Weak band at $1576 \mathrm{~cm}^{-1}$ is ascribed to the $\mathrm{C}=\mathrm{C}$ stretching. It is evident from the spectrum of TSA-AgNP, that the - $\mathrm{COO}^{-}$group is involved in the reduction of $\mathrm{Ag}+$ and - $\mathrm{SH}$ group of TSA is involved in the capping of AgNPs( presence of weak band around $2400 \mathrm{~cm}^{-1}$ ). Thus, the above observation provides direct evidence to the presence of an organic capping around silver nanoparticles.

X-ray photoelectron spectroscopy was used to study the change in the oxidation state of silver before and after the experimental treatments. The study was carried out with dry sample obtained from centrifuging colloidal silver solution. The spectral profile for the TSA derivatized AgNPs (Figure 7) shows two peaks at 368.54 and $374.64 \mathrm{eV}$ due to $\mathrm{Ag} 3 \mathrm{~d}_{5 / 2}$ and $\mathrm{Ag} 3 \mathrm{~d}_{3 / 2}$ orbital, respect-

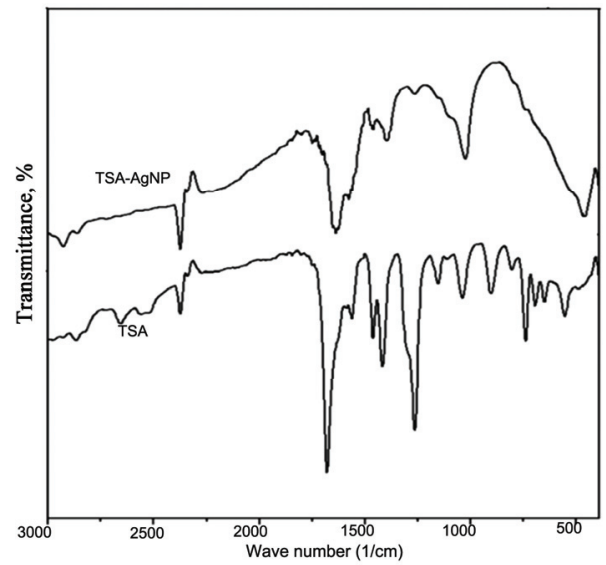

Figure 6. FTIR spectrum of free TSA and TSA capped AgNPs.

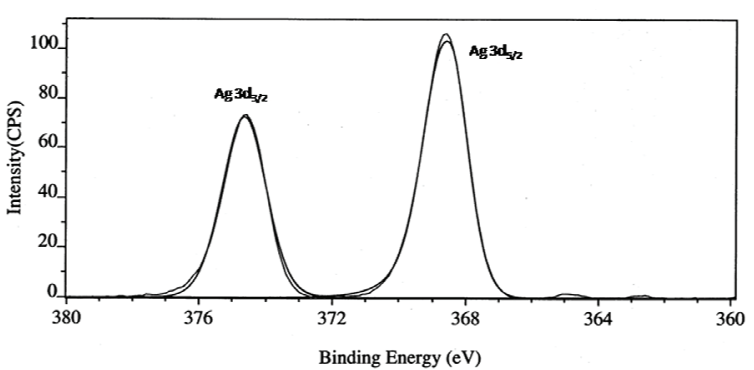

Figure 7. XPS spectrum of silver nanoparticles.

tively, which indicates that the silver present in the cluster is in the $\mathrm{Ag}(0)$ state.

The capping ability of TSA on the AgNPs was further substantiated from thermogravimetric analysis (TGA). Dry sample was utilized for this study. Figure 8 shows the thermogram for the synthesized TSA capped nanoparticles at thermal condition, which depicts continuous weight losses in the temperature region $100-800^{\circ} \mathrm{C}$. The dotted line in the figure shows the thermogram of TSA alone. A total weight loss of about $9.3 \%$ is indicative of presence of organic molecules. Hence, weight losses from the thermogram of AgNPs can be ascribed to the ready decomposition of TSA molecules from the surface of AgNPs. Therefore, the above observation apparently deduces the weakly bonding nature of TSA molecules on to the metal surface.

In conclusion, thiosalicylic acid under appropriate mole ratio to $\mathrm{Ag}$ ions serves as both reductant and stabilizer in the synthesis of silver nanoparticles in aqueous media. The rate of formation of the nanoparticles can be further enhanced by adopting different methodologies like photochemical and microwave dielectric heating. Among the three methods employed, microwave heating has been found to be advantageous in terms of energy saving, shorter processing time, uniformity of products, reduction of particle size and the nanoparticle stability.

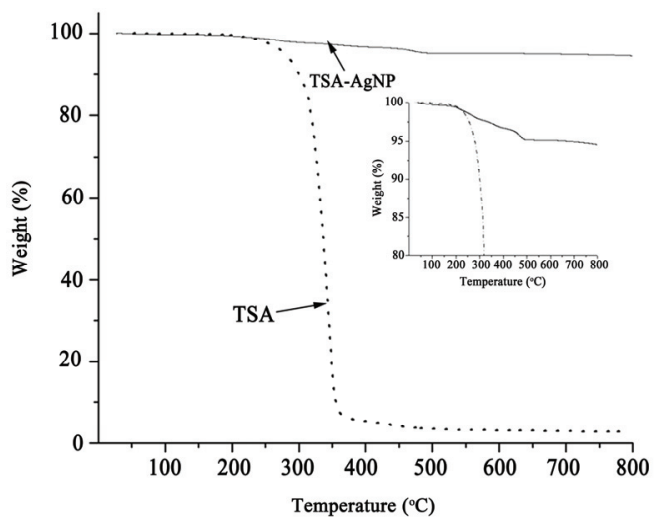

Figure 8. Thermogram of TSA and TSA capped AgNPs. Inset shows the thermogram in the $100-80 \%$ weight loss region under magnification. 
UV-vis spectroscopy confirms the presence of surface plasmon at $420 \mathrm{~nm}$, characteristic of $\mathrm{Ag}$ nanoparticles. Particle size measurement, zeta potential analysis and TEM results indicate that the silver nanoparticles synthesized by microwave irradiation have a size of less than 7 $\mathrm{nm}$ and are spherical. Stable silver sol, wherein attachment of thiosalicylic acid to $\mathrm{Ag}$ nanoparticle has been obtained as confirmed from the TGA and IR measurements. Unaltered particle morphology with long stability even after drying has also been obtained. All the three synthetic methodologies employed are simple, reproducible and could be adopted for direct one step synthesis and for achieving stable, monodisperse metal sols in mass

\section{Acknowledgements}

Dr B Sreedhar of Indian Institute of Chemical Technology, Hyderabad, India is thanked for the XPS measurements.

\section{REFERENCES}

[1] E. A. Chandross and R. D. Miller. "Nanostructures: Introduction," Chemical Reviews, Vol. 99, No. 7, 1999, pp. 1641-1642.

[2] L. M. Liz-Marzan, "Tailoring Surface Plasmons through the Morphology and Assembly of Metal Nanoparticles," Langmuir, Vol. 22, No. 1, 2005, pp. 32-41.

[3] Sampaio, K. C. Beverly and J. R. Heath, "DC Transport in Self-Assembled 2D Layers of Ag Nanoparticles," The Journal of Physical Chemistry B, Vol. 105, No. 37, 2001, pp. 8797-8800.

[4] M. Chen, Y.-G. Feng, X. Wang, T.-C. Li, J.-Y. Zhang and D.-J. Qian, "Silver Nanoparticles Capped by Oleylamine: Formation, Growth, and Self-Organization," Langmuir, Vol. 23, No. 10, 2007, pp. 5296-5304.

[5] X. Dong, X. Ji, H. Wu, L. Zhao, J. Li and W. Yang. "Shape Control of Silver Nanoparticles by Stepwise Citrate Reduction," The Journal of Physical Chemistry C, Vol. 113, No. 16, 2009, pp. 6573-6576.

[6] Y. Li, Y. Wu and B. S. Ong, "Facile Synthesis of Silver Nanoparticles Useful for Fabrication of High-Conductivity Elements for Printed Electronics," Journal of the American Chemical Society, Vol. 127, No. 10, 2005, pp. 3266-3267.

[7] A. D. McFarland and R. P. Van Duyne, "Single Silver Nanoparticles as Real-Time Optical Sensors with Zeptomole Sensitivity," Nano Letters, Vol. 3, No. 8, 2003, pp. 1057- 1062 .

[8] M. Muniz-Miranda, B. Pergolese, A. Bigotto and A. Giusti, "Stable and efficient silver substrates for SERS spectroscopy," Journal of Colloid and Interface Science, Vol. 314, No. 2, 2007, pp. 540-544.

[9] S. Sarkar, A. D. Jana, S. K. Samanta and G. Mostafa, "Facile synthesis of silver nano particles with highly efficient anti-microbial property," Polyhedron, Vol. 26, No. 15, 2007, pp. 4419-4426.

[10] D. G. Duff, A. Baiker and P. P. Edwards, "A new hydrosol of gold clusters. 1. Formation and particle size variation," Langmuir, Vol. 9, No. 9, 1993, pp. 2301-2309.

[11] W. Wang, S. Efrima and O. Regev, "Directing Oleate Stabilized Nanosized Silver Colloids into Organic Phases," Langmuir, Vol. 14, No. 3, 1998, pp. 602-610.

[12] V. V. Agrawal, G. U. Kulkarni and C. N. R. Rao, "Surfactant-promoted formation of fractal and dendritic nanostructures of gold and silver at the organic-aqueous interface," Journal of Colloid and Interface Science, Vol. 318, No. 2, 2008, pp. 501-506.

[13] H. -G. Liu, F. Xiao, C.-W. Wang, Q. Xue, X. Chen, Y.-I. Lee, J. Hao and J. Jiang, "Synthesis of one-dimensional silver oxide nanoparticle arrays and silver nanorods templated by Langmuir monolayers," Journal of Colloid and Interface Science, Vol. 314, No. 1, 2007, pp. 297303.

[14] A. Manna, T. Imae, M. Iida and N. Hisamatsu, "Formation of Silver Nanoparticles from a N-Hexadecylethylenediamine Silver Nitrate Complex," Langmuir, Vol. 17, No. 19, 2001, pp. 6000-6004.

[15] P. Mukherjee, A. Ahmad, D. Mandal, S. Senapati, S. R. Sainkar, M. I. Khan, R. Parishcha, P. V. Ajaykumar, M. Alam, R. Kumar and M. Sastry, "Fungus-Mediated Synthesis of Silver Nanoparticles and Their Immobiliza-tion in the Mycelial Matrix: A Novel Biological Approach to Nanoparticle Synthesis," Nano Letters, Vol. 1, 2001, pp. 515-519.

[16] W. Song, H. Jia, Q. Cong and B. Zhao, "Silver MicroFlowers and Large Spherical Particles: Controlled Preparation and Their Wetting Properties," Journal of Colloid and Interface Science, Vol. 311, No. 2, 2007, pp. 456-460.

[17] W. Wang and S. A. Asher, "Photochemical Incorporation of Silver Quantum Dots in Monodisperse Silica Colloids for Photonic Crystal Applications," Journal of the American Chemical Society, Vol. 123, No. 50, 2001, pp. 12528-12535.

[18] B. Xue, P. Chen, Q. Hong, J. Lin and K.L. Tan, "Growth of $\mathrm{Pd}, \mathrm{Pt}, \mathrm{Ag}$ and $\mathrm{Au}$ Nanoparticles on Carbon Nanotubes," Journal of Materials Chemistry, Vol. 11, No. 11, 2001, pp. 2378-2381.

[19] Z. Zhang, R. C. Patel, R. Kothari, C. P. Johnson, S. E. Friberg and P. A. Aikens, "Stable Silver Clusters and Nanoparticles Prepared in Polyacrylate and Inverse Micellar Solutions," The Journal of Physical Chemistry B, Vol. 104, No. 6, 2000, pp. 1176-1182.

[20] M. Zheng, M. Gu, Y. Jin and G. Jin, "Optical Properties of Silver-Dispersed PVP Thin Film," Materials Research Bulletin, Vol. 36, No. 5, pp. 853-859.

[21] J.-J. Zhu, X.-H. Liao, X.-N. Zhao and H.-Y. Chen, "Preparation of Silver Nanorods by Electrochemical Methods," Materials Letters, Vol. 49, No. 2, 2001, pp. 91-95.

[22] P. C. Lee and D. Meisel, "Adsorption and Surface- 
Enhanced Raman of Dyes on Silver and Gold Sols," The Journal of Physical Chemistry, Vol. 86, No. 17, 1982, pp. 3391-3395.

[23] B. Karthikeyan, "Novel Synthesis and Optical Properties of $\mathrm{Sm}^{3+}$ Doped Au-Polyvinyl Alcohol Nanocomposite Films," Chemical Physics Letters, Vol. 432, No. 4-6, 2006, pp. 513-517.

[24] P. K. Khanna, N. Singh, D. Kulkarni, S. Deshmukh, S. Charan and P. V. Adhyapak, "Water Based Simple Synthesis of Re-Dispersible Silver Nano-Particles," Materials Letters, Vol. 61, No. 16, 2007, pp. 3366-3370.

[25] M. Husein, E. Rodil and J. Vera, "Preparation of AgBr Nanoparticles in Microemulsions Via Reaction of $\mathrm{AgNO}_{3}$; with CTAB Counterion," Journal of Nanoparticle Research, Vol. 9, No. 5, 2007, pp. 787-796.

[26] K. Esumi, K. Matsuhisa and K. Torigoe, "Preparation of Rodlike Gold Particles by UV Irradiation Using Cationic Micelles as a Template," Langmuir, Vol. 11, No. 9, 1995, pp. 3285-3287.

[27] M. Y. Han and C. H. Quek, "Photochemical Synthesis in Formamide and Room-Temperature Coulomb Staircase Behavior of Size-Controlled Gold Nanoparticles," Langmuir, Vol. 16, No. 2, 1999, pp. 362-367.

[28] K. C. Grabar, R. G. Freeman, M. B. Hommer and M. J. Natan, "Preparation and Characterization of Au Colloid Monolayers," Analytical Chemistry, Vol. 67, No. 4, 1995, pp. 735-743.
[29] S. Komarneni, D. Li, B. Newalkar, H. Katsuki and A. S. Bhalla, "Microwave Polyol Process for Pt and Ag Nanoparticles," Langmuir, Vol. 18, No. 15, 2002, pp. 59595962.

[30] T. Yamamoto, H. Yin, Y. Wada, T. Kitamura, T. Sakata, H. Mori and S. Yanagida, "Morphology-Control in Microwave-Assisted Synthesis of Silver Particles in Aqueous Solutions," Bulletin of the Chemical Society of Japan, Vol. 77, No. 4, 2004, pp. 757-761.

[31] R. H. Ritchie, "Plasma Losses by Fast Electrons in Thin Films," Physical Review, Vol. 106, No. 5, 1957, pp. 874881.

[32] A. Kumar, H. Joshi, R. Pasricha, A. B. Mandale and M. Sastry, "Phase Transfer of Silver Nanoparticles from Aqueous to Organic Solutions Using Fatty Amine Molecules," Journal of Colloid and Interface Science, Vol. 264, No. 2, 2003, pp. 396-401.

[33] C. Petit, P. Lixon and M.P. Pileni, "In Situ Synthesis of Silver Nanocluster in AOT Reverse Micelles," The Journal of Physical Chemistry, Vol. 97, No. 49, 1993, pp. 12974-12983.

[34] P. Magudapathy, P. Gangopadhyay, B.K. Panigrahi, K. G. M. Nair and S. Dhara, "Electrical Transport Studies of Ag Nanoclusters Embedded in Glass Matrix," Physica B: Condensed Matter, Vol. 299, No. 1-2, 2001, pp. 142-146. 